\title{
Studying Membrane Topology of the Neuromuscular Junction by Electron Tomography of Thick Resin Slices in a Cs/Cc-corrected TEM
}

\author{
I.U. Wacker, ${ }^{*}$ I.V. Röder, ${ }^{* *}$ R. Rudolf, ${ }^{* *}$ S. Hillmer, ${ }^{* * *}$ B. Kabius, ${ }^{* * * *}$ G. Hofhaus****,***** \\ and R.R. Schröder***** \\ * Institut für Biologische Grenzflächen I, Karlsruher Institut für Technologie, CN, Germany \\ ** Institut für Toxikologie und Genetik, Karlsruher Institut für Technologie, CN, Germany \\ *** Zellbiologie, HIP, Universität Heidelberg, Germany \\ **** Argonne National Laboratory, Argonne, USA \\ ***** CellNetworks, BioQuant, Universität Heidelberg, Germany
}

Electron Tomography of whole cells or large volume tissue samples is of central importance for the imaging of macromolecular complexes in their native cellular environment. To overcome the tedious and technically difficult serial sectioning we wanted to image rather thick sections. So far imaging of thick samples was hampered by the effects of the large chromatic aberration (Cc) of TEMs. The dominant multiple inelastic scattering leads to blurred images, since electrons at different energies ("colours") will be imaged in different image planes. With the recent availability of novel, aberration-corrected TEMs (within the framework of the TEAM project [1]) we were able to record high-resolution tomograms from unusually thick sections of muscle tissue as a first biological application of this novel microscope.

In view of recent findings [2], that the molecular motor myosin is involved in recycling of the acetylcholine receptor at the nerve-muscle synapse (NMJ) a number of questions arose about the relationship between the topology of the synaptic membrane system and its function. In particular we are interested to visualize postsynaptic actin networks and a proposed membranous receptorrecycling compartment, where receptor storage and/or receptor quality control needs to be performed.

In conventional ultrathin sections of epon-embedded muscle material appearance of NMJs varies to a large extent (Fig. 1) from textbook-like synapses with rather short and straight postsynaptic folds (1A) over more branched structures (1B) to very convoluted membrane systems (1C), where internal and external space is hard to distinguish. To gain information about the 3D morphology we are using electron tomography of up to $1 \mu \mathrm{m}$ thick sections. Typical raw data from a tilt series $\left(+70^{\circ}\right.$ to $\left.-70^{\circ}\right)$ is shown in Fig. 2. Single sections from reconstructed 3D volumes (Fig. 3) illustrate the degree of intracellular detail obtainable. Postsynaptic folds often assume a tooth-like morphology, with obvious omega-shaped membrane invaginations (arrows) and a distinctive, cavity-like morphology in certain areas of the post-synaptic membrane. A typical slice through a reconstructed synaptic bouton with the corresponding segmentation (Fig. 4) demonstrates the considerable extension of in depth information content achievable. At present we analyze the post-synaptic fold structure. Preliminary results show that an extended network of compartments is visualized.

References

[1] B. Kabius et al., J. Electron Microsc. 58 (2009) 147.

[2] I.V. Röder et al., Proc. Nat. Acad. Sci. USA 107 (2010) 2031-2036.

[3] Part of this work was done at the Argonne National Laboratory as part of the TEAM project. 

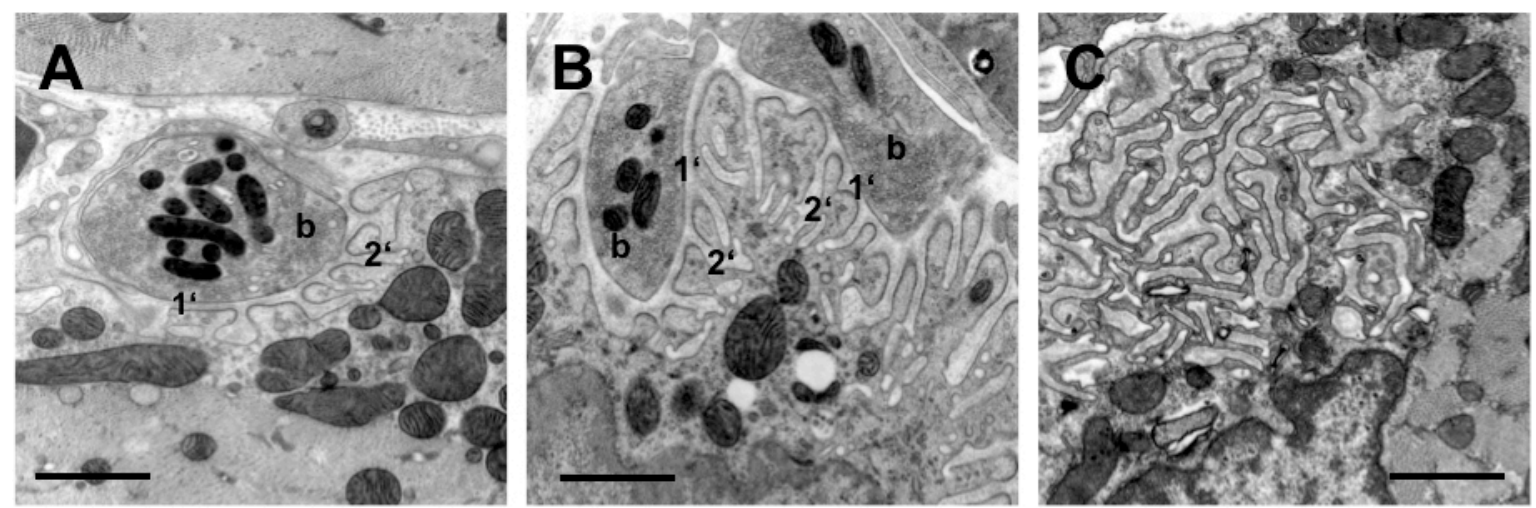

Fig. 1 Variable appearance of NMJ in 70nm sections of A,B) mouse diaphragms after high pressure freezing and freeze substitution. C) mouse tibialis anterior muscle after chemical fixation, all embedded in Epon. b synaptic bouton, 1' primary, 2' secondary synaptic cleft. Scale bars $1 \mu \mathrm{m}$.
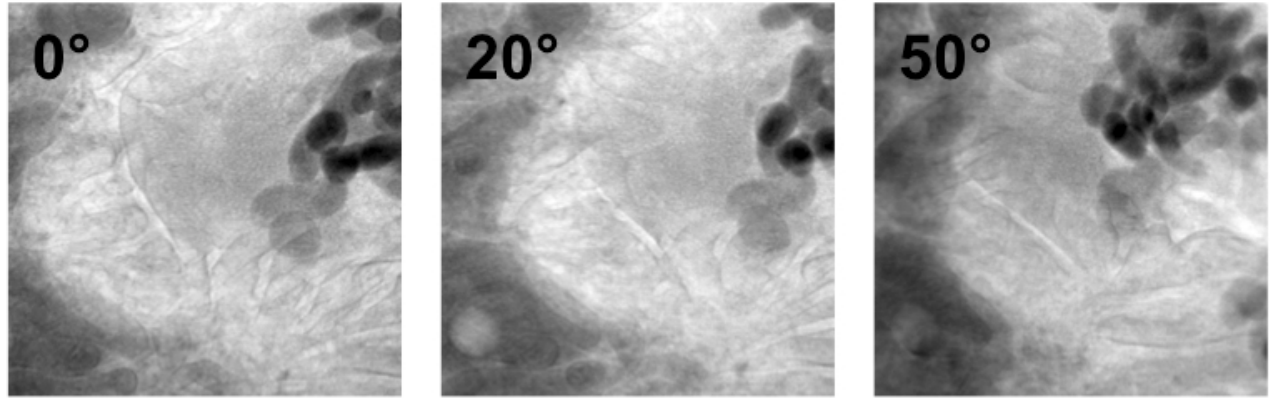

Fig. 2 Raw data from tilt series: $1 \mu \mathrm{m}$ thick section recorded at $300 \mathrm{keV}$ electron energy (Cs/Cc corr).
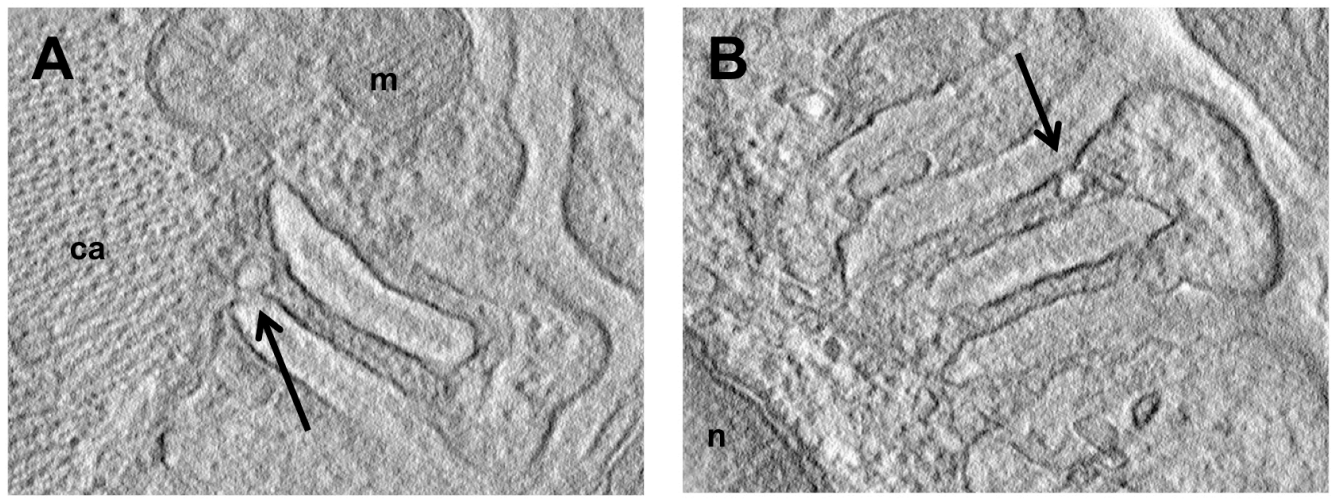

Fig. 3 Slices from tomographic reconstructions: 600nm thick sections (chemical fixation) recorded at $80 \mathrm{keV}$ electron energy, tilt was $+30^{\circ}$ to $-30^{\circ}$. ca contractile apparatus, $\mathrm{m}$ mitochondria, $\mathrm{n}$ nucleus.
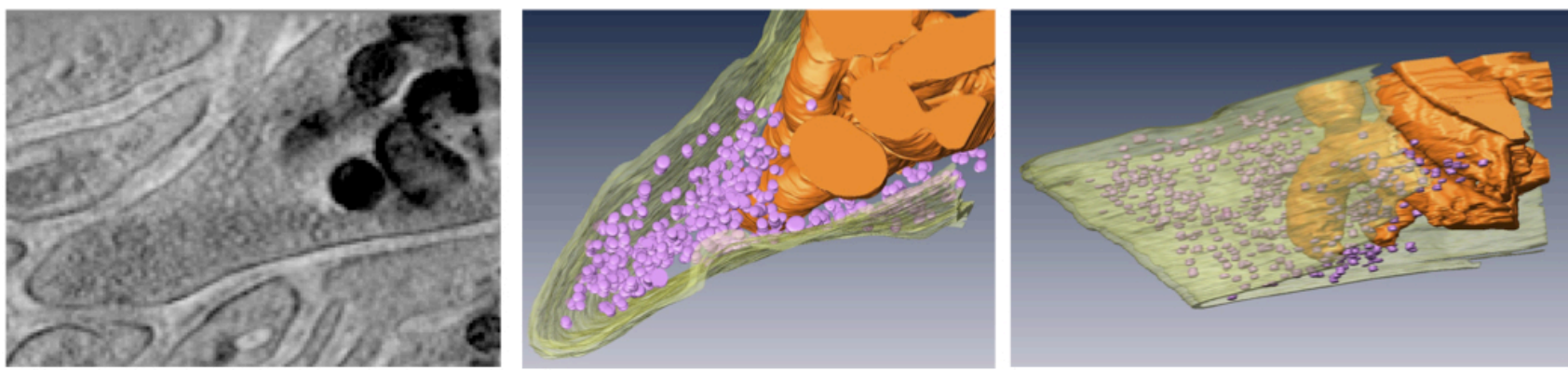

Fig. 4 Slice from 3D reconstruction of tilt series in Fig. 2 and segmentation of synaptic bouton (cryo fixation). Presynaptic plasma membrane yellow, mitochondria orange, synaptic vesicles magenta. 